\title{
Short-term recall of paired-associates as a function of the number of interpolated pairs'
}

\author{
J. W. BRELSFORD, JR., L. KELLER, R. M. SHIFFRIN AND R. C. ATKINSON \\ STANFORD UNIVERSITY
}

A continuous technique for studying short-term memory of paired-associates was used. For 4, 6, or 8 stimuli, recall was found to be a decreasing function of the number of pairs of items interpolated between study and test on a given item. Reliable differences between the functions for 4,6 , and 8 stimuli were found.

In a typical paired-associates paradigm for the study of short-term memory, a list of items is presented and the $S$ is then required to give the correct response to one of the stimuli selected from the list (e.g., Murdock, 1963). Since only one of the pairs is tested on a given presentation of the entire list, it is difficult to gather large amounts of data under homogeneous conditions. This paper introduces a modification of this short-term memory procedure that provides for the efficient gathering of large amounts of data under fairly homogeneous conditions. The technique is similar to one used by Yntema \& Mueser (1962), and permits us to collect two to three thousand comparable observations from a $S$ in $101-$ hr. sessions.

METHOD

Subjects. The Ss were 10 students from Stanford University who received $\$ 2$ an hour. Each S participated in at least $101-\mathrm{hr}$. experimental sessions.

Apparatus. All programming, generation of stimuli, and response recording were carried out with an on-line PDP-1 computer. Stimuli were electronically generated and displayed on the face of a cathode ray tube (CRT). Responses were made on an electric typewriter keyboard located immediately below the lower edge of the CRT.

Stimuli and Responses. The stimuli were two-digit numbers randomly selected for each session from a set of all two-digit numbers between 00 and 99. Once a set of stimuli was selected for a given session, it was used throughout that session. In each session the same six responses were used: the letters $F, G, H, J$, $K$, and $\mathrm{L}$.

Procedure. For each session each $\mathrm{S}$ was assigned to one of three experimental conditions. The conditions (labeled 4,6 , or 8 ) indicate the number of stimuli that were used in a given session. An attempt was made to assign Ss to each condition once in each consecutive three-session block. Each session began with a series of consecutive study trials: one study trial for each stimulus to be used in the session. On each study trial the word study appeared on the upper face of the CRT. Beneath the word study thereappeared one of the stimuli to be used in the session along with a randomly-selected response member. Ss had been initially instructed to try to remember the association between the pairs of items which appeared along with the word study. Each of these initial study trials lasted for 3 sec., with a 3-sec. intertrial interval (ITI). As soon as there had been one initial study trial for each stimulus to be used in the session, the session proper began.

Each trial involved a fixed series of events: (1) The word test appeared on the upper face of the CRT. Beneath the word test there appeared a randomly selected member of the stimulus set. Ss had been instructed that, when the word test and a stimulus appeared on the CRT, they were to respond with the last item they had associated with that stimulus. This test portion of each trial lasted for 3 sec. (2) CRT was blacked out for 2 sec. (3) The word study appeared on the upper face of the CRT for 3 sec. Below the word study a stimulus-response pair appeared. The stimulus was the same that had been used in the preceding test portion of the trial. The response item was randomly selected from the response set, with the stipulation that the response be different from the immediately preceding response item assigned to that stimulus. (4) Three-sec. ITI before the next trial.

\section{RESULTS}

Figure 1 presents the proportion of correct responses in 50-trial blocks, for each of the three stimulus conditions. It can be seen that the curves appear

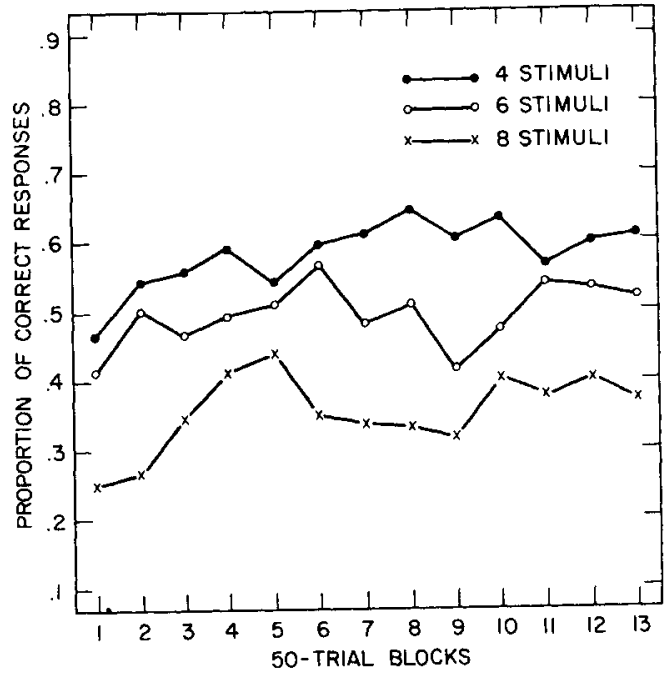

Fig. 1. Proportion of correct responses as a function of trial blocks. Data for each stimulus condition are summed over all subjects and sessions. 
to have leveled off at three distinct asymptotes. Due to the warm-up effect, subsequent analyses will not include any of the first-day sessions nor the first 25 trials of subsequent daily sessions. The number of trials intervening between study and test for a given item will be referred to as the lag for that item. Thus, if the test for a given stimulus occurs immediately following the study for that stimulus, the lag is 0 . If one trial intervenes, the lag is 1 , and so on. Figure 2 presents the proportion of correct responses as a function of lag. In order to determine the reliability of the differences between the three curves in Fig. 2 the proportions correct for each $\mathrm{S}$ for each of the conditions were calculated and then ranked. An analysis of variance for correlated means indicated that the curves differed reliably $(\mathrm{F}=7.65, \mathrm{df}=2 / 18, \mathrm{p}<.01)$.

\section{DISCUSSION}

For a given lag, the more stimuli used, the more different intervening stimuli there are. For example, with a lag of 3 it is much more likely that the three intervening stimuli are all different when there are eight stimuli than when there are four stimuli. Excepi for lag 1, it might be the case that the differences in the three curves merely reflect the number of different intervening items for a given lag. In an additional analysis, the curves in Fig. 2 were replotted in such a way that there were no repeated stimuli for a given lag.

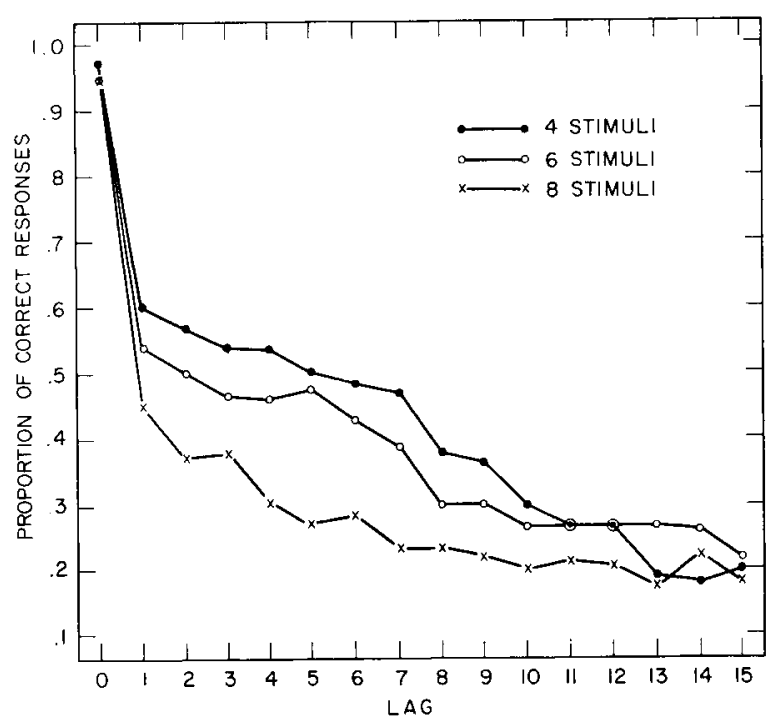

Fig. 2. Proportion of correct responses as a function of the lag between study and test trials. Data for each stimulus condition are summed over all subjects and sessions.
These curves were found to be even more divergent than those in Fig. 2. It thus appears that the differences in the three curves are not simply due to an artifact of the presentation procedure.

In a recent theoretical formulation of short- and long-term memory, Atkinson \& Shiffrin (1965) proposed a model that may be applied to the present experiment. The central assumption is that items enter a constantsized memory buffer knocking out an item from the buffer as they do so. The present results can be explained qualitatively in this framework if it is assumed that an item presented for test, which is currently in the buffer, replaces itself rather than another item. In the conditions with fewer stimuli, items will be replacing themselves more often and forgetting will be slower. This model predicts the qualitative aspects of the data in Fig. 2 such as the divergence and then convergence of the curves to the guessing level. It remains for a more detailed analysis to determine just how well a model of this sort accounts quantitatively for the data.

Some additional remarks should be made regarding the relation of the results in Figs. 1 and 2. It is possible that all three curves in Fig. 2 could be identical, and still generate differences of the type displayed in Fig. 1 among the three groups. That is, the probability of a correct response as a function of lag could be independent of the size of the stimulus set, and still we would obtain differences in the overall rate of correct responding since smaller set sizes would tend to have shorter lags for testing. Thus the major findings in this study are summarized by the functions in Fig. 2 .

One final point should be brought out in connection with the present data. By the time 10 or more pairs of items have intervened between the study and test for a given item, Ss are performing at close to chance level. It thus seems that a short-term memory process is the dominant factor in the present experimental situation.

\section{References}

Atkinson, R. C., \& Shiffin, R. M. Mathematical models for memory and learning. Tech. Rep. 79, Institute for Mathematical Studies in the Social Sciences, Stanford Univer., 1965.

Murdock, B. B., Jr. Short-term retention of single paired associates. J. exp. Psychol., 1963, 65, 433-443.

Yntema, D. B., \& Mueser, G. E. Keeping track of variables that have few or many states. J. exp. Psychol., 1962, 63, 391-395.

\section{Note}

1. This study was supported in part by the National Aeronautics and Space Administration, Grant No. NGR-05-020-036. 